\title{
Monocular 3D Vision Using Real-Time Generated Scene with Depth of Field Effect
}

\author{
Takashi Hosomi and Kunio Sakamoto \\ Department of Intelligence and Informatics, Konan University \\ 8-9-1 Okamoto, Higashinada, Kobe 658-8501, Japan
}

\begin{abstract}
The human vision system has visual functions for viewing 3D images with a correct depth. These functions are called accommodation, vergence and binocular stereopsis. Most 3D display system utilizes binocular stereopsis. The authors have developed a monocular 3D vision system with accommodation mechanism, which is useful function for perceiving depth.
\end{abstract}

Keywords: monocular stereoscopic display, real-time stereogram, 3-D display.

\section{Concept}

To realize natural 3D viewing, we have developed the monocular vision system, which can directly project stereoscopic image on a retina, and a 3D image generation system, which can make 3D computer graphics in accordance with accommodation. Assume that an actual object is in the real world. When you perceive this object, a part of the projected image on a retina might be a blur by the lens of an eye. It is a physiological response called as accommodation. In case of virtual 3D image viewing as shown in Fig. 1, you can watch a correct 3D image as the actual object is in there if the projected retina image has appropriate blur in compliance with focus adjustment of your eye. Then you might perceive virtual images with same accommodation as you watch real objects. Thus a monocular 3D vision system can provide correct 3D viewing with accommodation, vergence and binocular stereopsis and without a tired feeling at long time watching when the retina image is directly projected and external stimulation induces the focus adjustment by changing the thickness of an eye lens.

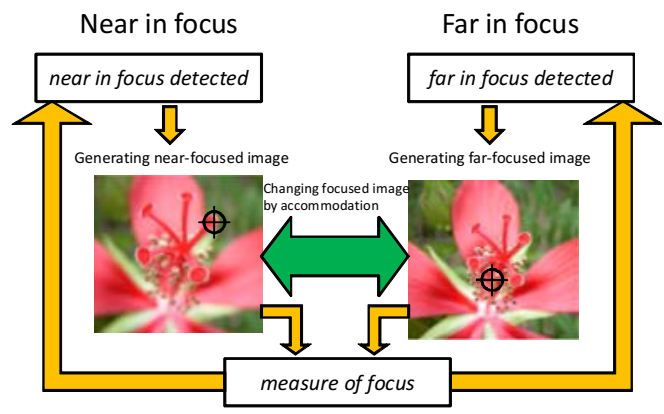

Fig. 1. Flow of generating focused image 


\section{Display System}

Fig. 2 shows the principle of a reconstruction of the 3D image by the stereo-viewing. Fig. 2 (a) shows the optical configuration of a conventional stereogram. To display a point object $P$, observers turn on a point $P_{L}$ for the left eye and a point $P_{R}$ for the right eye as shown in this figure. The observer perceives that a point object exists on a spatial position $\mathrm{P}$ due to the binocular parallax. Fig. 2 (b) shows the optical configuration of a monocular multi-view stereogram. To display a point object $\mathrm{P}$, observers turn on points $\mathrm{P}_{\mathrm{R} 1}$ and $\mathrm{P}_{\mathrm{R} 2}$ for the right eye as shown in this figure. At the monocular multi-viewing, the observer adjusts the focal length of an eye to match with the spatial position $\mathrm{P}$, then projected images of pixels $\mathrm{P}_{\mathrm{R} 1}$ and $\mathrm{P}_{\mathrm{R} 2}$ are focused to the same position on the retina. So the observer naturally perceives that a point object exists on a spatial position $\mathrm{P}$ due to the monocular parallax. Fig. 3 shows the principle of the 3D vision system using monocular stereoscopy. This display system consists of an LCD panel, an acrylic plate and an optical lens. The observers perceive parallax images at the just point, which the optical lens converges the light on. To perceive multiple parallax images with just one eye, the image shifting optics consists of a parallel plane acrylic plate, whose inclination causes the image to shift as shown in Fig. 3. An LCD panel is used as the displaying plane of parallax images. The signal controller sends an image signal to the LCD panel to the tune of a control signal. Then the displaying plane of parallax images creates monocular multi-viewing images.

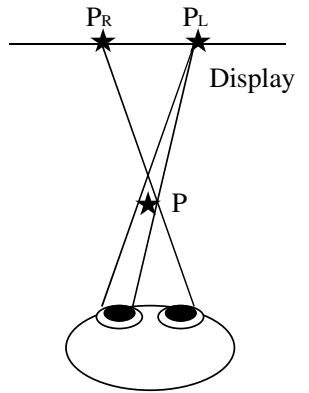

(a)

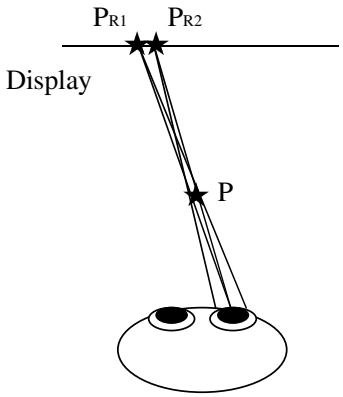

(b)

Fig. 2. The principle of a stereogram

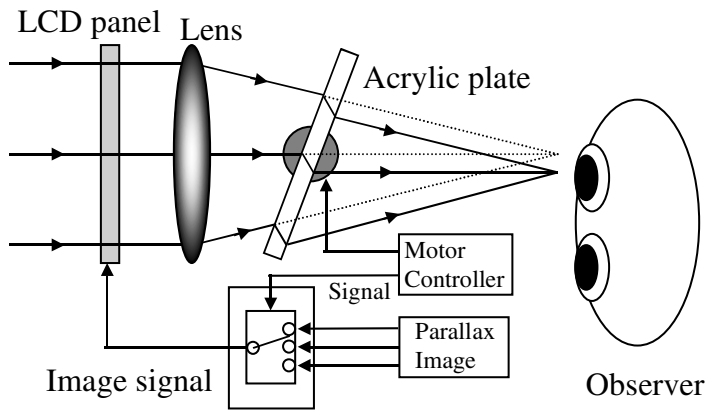

Signal controller

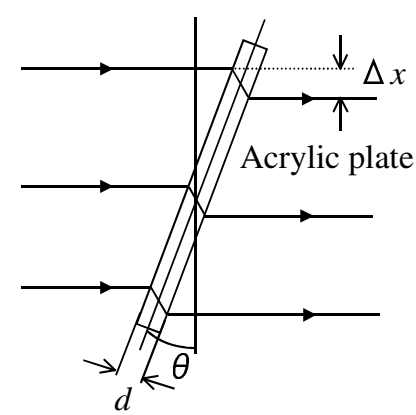

Fig. 3. Optical layout of the monocular 3-D display 